Pacific

Journal of

Mathematics

\title{
ON AMBIENTAL BORDISM
}

CARLOS Biasi

Volume 163 No. 1

March 1994 


\title{
ON AMBIENTAL BORDISM
}

\author{
Carlos Biasi
}

Let $M^{m}$ be a closed and oriented submanifold of a closed or oriented manifold $N^{n}$, such that $[M, i]=0 \in \Omega_{m}(N)$, where $i: M \rightarrow N$ is the inclusion and $\Omega_{m}(N)$ is the $m$ th oriented bordism group of $N$. If $n=m+2$ or $m \leq 3$ or $m \leq 4$ and $n \neq 7$ then $M$ bounds in $N$.

Introduction. Let us consider $M^{m}$ a closed submanifold of $N^{n}$. In this paper, we study the possibility that there exists submanifold $W^{m+1} \subset N^{n}$ such that $\partial W=M$. If $M=S^{m}$ and $N=S^{m+2}$, such that a submanifold $W$ is called a Seifert surface knot $S^{m}$. In [5], Sato showed that every connected closed and oriented submanifold $M^{m}$ of $S^{m+2}$ is a boundary of an oriented surface of $S^{m+2}$.

In [4], Hirsch studies the following problem: If a compact connected and oriented manifold $M^{m}$ bounds, does there exist embedding from $M^{m}$ into $\mathbb{R}^{n}$ which is a boundary in $\mathbb{R}^{n}$ ?

The answer is yes, if $n \geq 2 m$.

The difference between the two problems is that, in our case, the embedding from $M$ into $N$ is fixed.

There is an obvious necessary condition for the existence of $W$, when $M$ and $N$ are oriented manifolds.

Let $\Omega_{m}(N)$ be the $m$ th oriented bordism group of $N$ [2]. If $i: M \rightarrow N$ is the inclusion map, we can define an element $[M, i]$ in $\Omega_{m}(N)$ and see that $[M, i]=0$ if $M$ bounds in $N$.

Generally, the converse in not true, but sometimes the vanishing of [ $M, i]$ guarantees the existence of $W$, for example if the codimension $n-m$ is large.

We prove the following theorem.

THeOREM 5.2. Let us suppose that $M^{m} \subset N^{n}, n>m+1$, is such that $[M, i]=0$ in $\Omega_{m}(N)$. Then $M$ bounds in $N$ if one of the following conditions occurs:

(a) $n=m+2$,

(b) $m \leq 3$,

(c) $m \leq 4$ and $n \neq 7$. 
In his Doctoral thesis [1] the author proved that, when $n=2 m+$ 1 , and $M$ and $N$ are closed and oriented, a submanifold $M \subset N$ bounds in $N$ if, and only if, $[M, i]=0 \in \Omega_{m}(N)$.

\section{A more general problem of ambiental bordism. Let}

$$
G \subset O(n-m-1), \quad n>m+1,
$$

be a closed transformation group and let $\gamma_{G} \rightarrow B G$ be the classifying fiber bundle of $(n-m-1)$-vector bundles which have a $G$-structure.

Let us consider $M G$ the Thom space of $\gamma_{G}$. We have:

$$
\pi_{i}(M G)= \begin{cases}0, & i<n-m-1, \\ \mathbb{Z}, & i=n-m-1 \text { and } G \subset \mathrm{SO}(n-m-1), \\ \mathbb{Z}_{2}, & i=n-m-1 \text { and } G \not \subset \mathrm{SO}(n-m-1) .\end{cases}
$$

Let us consider now $N^{n}$ to be a closed connected manifold which we assume to be oriented if $G \subset \mathrm{SO}(n-m-1)$. (If $G \not \subset \mathrm{SO}(n-m-1$ ) we drop the orientability hypothesis.)

Let $M^{m} \subset N^{n}$ be a closed submanifold and let us suppose that the normal fiber bundle $\nu_{M}$ of $M$ in $N$ has a cross section $s$, nowhere zero, such that $\nu_{M}=\{s\} \oplus \xi$, where $\{s\}$ is a subbundle generated by $s$ and $\xi$ is a $(n-m-1)$-vector bundle endowed with a $G$-structure.

We shall say that a submanifold $W \subset N$ satisfies condition (*) if it has the properties:

(i) $\partial W=M$ and $s$ is the inward-pointing vector field on $\partial W$.

(ii) the normal fiber bundle $\nu_{W}$ has a $G$-structure which agrees with the given $G$-structure of $\xi$ over $M$. (Observe that $\xi=\nu_{W} \mid M$.)

2. Primary obstruction to the existence of $W$. Let $V$ be a closed tabular neighborhood of $M$ in $N, A=\partial W$ and $X=N-\stackrel{\circ}{V}$. We can think $s$ a function $s: M \rightarrow A$. Then $s(M)$ is a submanifold of $A$, whose normal fiber bundle is isomorphic to $\xi$. By the Thom construction there exists a function $f: A \rightarrow M G$ such that, if $\infty$ is the point at infinity to $M G$, then $f$ is differentiable on $A-f^{-1}(\infty)$, $f$ is transversal to $B G$ and $f^{-1}(B G)=(M)$ [6].

We shall take $\pi_{m-n-1}(M G)$ as the cohomology coefficient group. Let $e \in H^{n-m-1}(M G)$ be the fundamental class of the space $M G$. We know that $f^{*}(e)=\alpha$, where $\alpha$ is the dual class of $s_{*}\left(\mu_{M}\right)$ and $\mu_{M}$ is the fundamental class of $M$.

If $f: A \rightarrow M G$ extends to a map $\bar{f}: X \rightarrow M G$, then we can suppose, up to homotopy, that $\bar{f}$ is differentiable in $X-\bar{f}^{-1}(\infty)$ and that $\bar{f}$ is transversal to $B G$. Taking $W_{1}=\bar{f}^{-1}(B G)$ we obtain a submanifold of $X$ whose boundary is $s(M)$. 
Let us observe that this submanifold can be extended to a submanifold $W$ which satisfies condition $(*)$.

We conclude then that there exists $W$, satisfying $(*)$, if and only if $f$ extends to $X$.

The class $\delta f^{*}(e)$ is the obstruction to the extension of $f$ to the $(n-m)$-skeleton of $X$, where $\delta: H^{n-m-1}(A) \rightarrow H^{n-m}(X, A)$ is the coboundary operator.

Consider the commutative diagram:

$$
\begin{array}{ccc}
H^{n-m-1}(A) \stackrel{\delta}{\longrightarrow} & H^{n-m}(X, A) \\
\downarrow_{D} & & \downarrow D \\
H_{m}(A) \stackrel{s_{*}}{\longrightarrow} H_{m}(X) \cong H_{m}(N-M) .
\end{array}
$$

We conclude that the primary obstruction to the extension of $f$, up to duality, is the element $s_{*}\left(\mu_{M}\right) \in H_{m}(N-M)$ (regarding $s$ as function from $M$ into $N-M$ ).

Hence, we have:

Proposition 2.1. $f$ extended to the $(n-m)$-skeleton of $X$ if, and only if, $s_{*}\left(\mu_{M}\right)=0$ in $H_{m}(N-M)$.

Assuming that $s_{*}\left(\mu_{M}\right)=0$, let us consider two cases:

1. $G=O(n-m-1)$.

Here, $f$ extends up to the $(n-m+1)$-skeleton of $X$, because $\pi_{n-m}(M G)=0$ and, if $n-m=2$, then $f$ extends to all of $X$ since $M O(1)$ is a $K\left(\mathbb{Z}_{2}, 1\right)$ space.

2. $G=\mathrm{SO}(n-m-1)$.

Since $\pi_{n-m+i}(M G)=0, i=0,1,2, f$ extends up to the $(n-m+3)$-skeleton of $X$. Hence, if $\operatorname{dim} M \leq 3, f$ extends.

On the other hand, if $n-m=2$ or 3 then $M G$ is a $K(\mathbb{Z}, 1)$ or $K(\mathbb{Z}, 2)$, respectively. In any case, $f$ extends globally.

3. Oriented ambiental bordism. From now on, all manifolds and submanifolds will be considered to be oriented.

THEOREM 3.1. Let us suppose that:

(a) $H_{j}(X)=0,0<j<m-3$.

(b) The canonical homomorphism $\pi_{n-1}(\operatorname{MSO}(n-m-1)) \stackrel{\varphi}{\longrightarrow} \Omega_{m}$ is injective.

There exists $W$ satisfying (*) if, and only if, $s_{*}\left(\mu_{M}\right)=0 \in H_{m}(X)$ and $M$ is a boundary. 
Proof. Let us use the notation $\pi_{i}=\pi_{i}(\mathrm{MSO}(n-m-1))$. If $s_{*}\left(\mu_{M}\right)=0$, then $f$ extends to the $(n-m)$-skeleton of $X$.

From hypothesis (a) and Lefschetz duality, we conclude that

$$
H^{j}\left(X, A, \pi_{j-1}\right)=0, \quad n-m<j<n .
$$

Let $D$ be an open disk of $X-A$. Since $X$ is orientable, $H^{j}\left(X-D, A, \pi_{j-1}\right) \cong H^{j}\left(X, A, \pi_{j-1}\right)=0, n-m<j<n$. Hence, there exists an extension $\bar{f}: X-D \rightarrow Y$ of $f: A \rightarrow Y$, where $Y=\operatorname{MSO}(n-m-1)$.

Let us consider $S=\partial D$ and $h=\bar{f} \mid \partial D: S \rightarrow Y$. We may suppose that $h$ is transversal to $\operatorname{BSO}(n-m-1)$ and let

$$
M^{m}=h^{-1}(\operatorname{BSO}(n-m-1)) \text {. }
$$

Consider $\bar{W}=\bar{f}^{-1}(\operatorname{BSO}(n-m-1))$, a bordism between $M_{1}$ and $s(M)$. Since $s(M)$ is a boundary, $M_{1}$ also is.

We have also that $\psi([h])=\left[M_{1}\right]=0$ and since $\psi$ is a monomorphism, $h$ is homotopic to a constant map and so $h$ extends over D.

The converse is straightforward.

4. On the existence of normal vector fields homologous to zero in $N-M$. In the next section, we show that in certain situations it is possible to obtain a cross-section $s: M \rightarrow S\left(\nu_{M}\right)$ such that $s_{*}\left(\mu_{M}\right)=$ $0 \in H_{m}(N-M)$, where $S\left(\nu_{M}\right) \rightarrow M$ is the normal sphere bundle of $M$ in $N$.

Proposition 4.1. The Euler class of the normal bundle of $M^{m}$ in $N^{n}$ is zero if and only if $i_{*}\left(\mu_{M}\right) \subset \operatorname{im} j_{*}$, where $\mu_{M}$ is the fundamental class of $M$ and $i: M \rightarrow N, j: N-M \rightarrow N$ are inclusion maps.

Proof. Let us consider $e \in H^{n-m}(M, \mathbb{Z})$, the Euler class of the normal bundle $\nu_{M}$, and let $D_{A}: H^{n-m}(M: \mathbb{Z}) \rightarrow H_{m}(N, N-M ; \mathbb{Z})$ be the Alexander duality. We have that $D_{A}(e)=\alpha_{*}\left(\mu_{M}\right)$ where $\alpha_{*}$ is induced by the inclusion map $\alpha:(N, N-M)$.

Using the exact sequence of pair $(N, N-M)$ it follows that $\alpha_{*}\left(\mu_{M}\right)$ $=0$ if, and only if, $i_{*}\left(\mu_{M}\right) \subset \operatorname{im} j_{*}$.

COROLlARY 4.2. If $M^{m} \subset N^{n}$ is homologous to zero, $n-m=2$ or $n \geq 2 m$, then $M$ has a normal vector field that is nowhere zero.

Proof. By Proposition 4.1 the Euler class of $\nu_{M}$ is zero. Then there is a nowhere zero normal vector field on the $(n-m)$-skeleton 
of $M$, which can be extended to all $M$, because $n-m \geq m$ or $\pi_{i}\left(R^{2}-0\right)=0, i>1$ in the case $n-m=2$.

Let $\pi: E \rightarrow M^{m}$ be a differentiable $\mathrm{SO}(n+1)$-bundle with fiber $S^{n}$ and base $M^{m}$ (and oriented manifold).

If $s: M \rightarrow E$ is a cross-section, let $\theta_{s}$ be the Poincare dual to $\bar{s}_{*}\left(\mu_{M}\right)$, where $\bar{s}=-s$ is the opposite cross-section to $s$.

Having fixed a cross-section $s_{0}: M \rightarrow E$, the following diagrams are commutative:

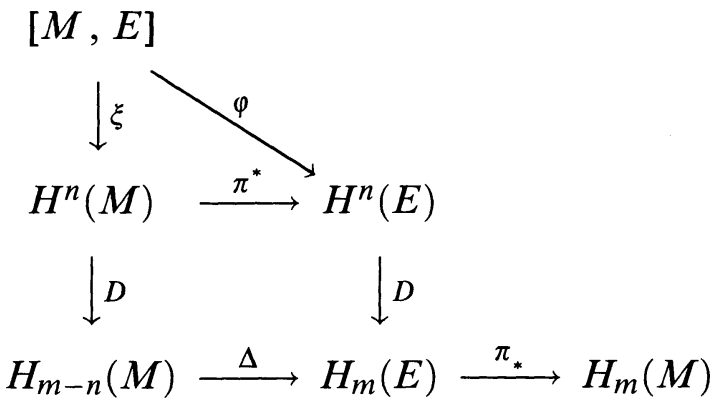

where $[M, E]$ is the set of homotopy classes of cross-sections, $\xi([s])=$ $\bar{s}^{*}\left(\theta_{\bar{s}_{0}}\right) ; \varphi([s])=\theta_{\bar{s}_{0}}-\theta_{\bar{s}}$, is Poincaré duality and last line is a portion of the generalized Gysin sequence.

We define $\psi:[M, E] \rightarrow H_{m}(E)$ by $\psi([s])=s_{s_{*}}\left(\mu_{M}\right)-s_{*}\left(\mu_{M}\right)$ and observe that $\psi=D \circ \psi$.

If $m \leq n+1$ or $n=1$, then the function $\xi$ is onto and so the image of $\psi$ is the kernel of $\pi_{*}$.

This fact will be applied in the proof of Proposition 4.3 below, where the fiber bundle to be considered is $S\left(\nu_{M}\right) \rightarrow M$.

Proposition 4.3. Let $M^{m} \subset N^{n}, n=m+2$ or $n \geq 2 m$, be an oriented submanifold homologous to zero in an oriented manifold $N$. Then there exists a cross-section $r: M \rightarrow S\left(\nu_{M}\right)$ such that its image is homologous to zero in $H_{m}(N-m)$.

Proof. Let $s_{0}: M \rightarrow S\left(\nu_{M}\right)$ be a cross-section that is nowhere zero (Corollary 4.2) and let us consider the commutative diagrams:

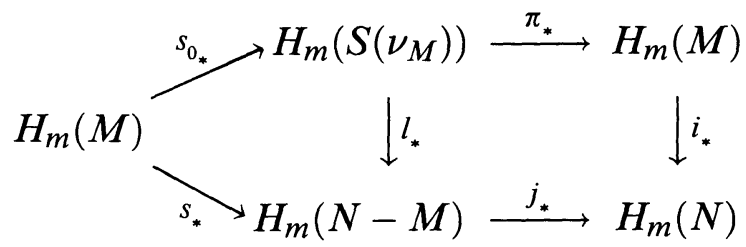

where $s_{*}=l_{*}\left(s_{0_{*}}\right)$ and $l_{*}$ is induced by the inclusion $S\left(\nu_{M}\right) \rightarrow$ $(N-M)$. 
We have $j_{*} s_{*}\left(\mu_{M}\right)=i_{*} \pi_{*} s_{0}\left(\mu_{M}\right)=0$ implying that $s_{*}\left(\mu_{M}\right)$ belongs to the kernel of $j_{*}$ which is the image of $\partial: H_{m+1}(N, N-M) \rightarrow$ $H_{m}(N-M)$.

Let us consider the following commutative diagram:

$$
\begin{array}{ccc}
H_{m+1}\left(D\left(\nu_{M}\right), S\left(\nu_{M}\right)\right) & \partial & H_{m}\left(S\left(\nu_{M}\right)\right) \\
\downarrow_{\text {exc }} & & \downarrow_{*} \\
H_{m=1}(N, N-M) & \stackrel{\partial}{\longrightarrow} & H_{m}(N-M) .
\end{array}
$$

It follows that there exists an element $\mu \in H_{m}\left(S\left(\nu_{M}\right)\right)$ such that $\mu \in \operatorname{Ker} \pi_{*}$ and $j_{*}=s_{*}\left(\mu_{M}\right)$.

Since $\operatorname{im} \psi=\operatorname{ker} \pi_{*}$, there exists a cross-section $r: M \rightarrow S\left(\nu_{M}\right)$ such that $\psi([r])=\mu$.

But $\psi([r])=s_{0}\left(\mu_{M}\right) \rightarrow r_{*}\left(\mu_{M}\right)$ so $j_{*} r_{*}\left(\mu_{M}\right)=0$ in $H_{m}(N-M)$. Hence, the image of $r: M \rightarrow S\left(\nu_{M}\right)$ is homologous to zero in $N-M$.

5. A theorem on ambiental bordism. Let us consider $\Omega_{j}(N)$ to be the $j$ th bordism group of $N$.

If $H_{j}(N)=0,0<j<m-3$, it is possible using the bordism spectual sequence [2] to show that the function $\Omega_{m}(N) \rightarrow H_{m}(N) \oplus$ $\Omega_{m}$, which associates to each pair $[M, f]$ the element $\mu([M, f])+$ $[M]$, is an isomorphism, where $\mu$ is the canonical homomorphism.

In the proof of Theorem 5.2, we are going to use the following lemma, which has been proved in [1] (the proof, if $q>m$, is due to Thom [6]).

LEMMA 5.1. The homomorphism $\varphi: \pi_{q+m}(\operatorname{MSO}(q)) \rightarrow \Omega_{m}, q \geq$ $m$, is an isomorphism.

THEOREM 5.2. Let us suppose $M^{m} \subset N^{n}, n>m+1$, is such that $[M, i]=0$ in $\Omega_{m}(N)$. Then $M$ bounds in $N$ if one of the following conditions occurs:

(a) $n=m+2$,

(b) $m \leq 3$,

(c) $m \leq 4$ and $n \neq 7$.

Proof. Any one of the conditions (a), (b) and (c), based on previous results, imply that normal bundle $\nu_{M}$ has a cross-section nowhere zero such that, considering $s$ as a function from $M$ into $N-M$, $s_{*}\left(\mu_{M}\right)=0 \in H_{m}(N-M)$. 
If (a) or (b) occurs, the theorem follows from case 2, already discussed in $\S 2$

If $n=4$ and $n \geq 8$, we apply Theorem 3.1 .

REMARK 1. If $n=m+2$ or $m \leq 3$, then $[M, i]=0 \in \Omega_{m}(N)$ if, and only if, $M$ is homologous to zero in $N$.

REMARK 2. When $m=4$ and $n \neq 7$, although we shall prove that $[M, i]=0$ implies the existence of a normal section nowhere zero (Th. 5.3) we are not able to prove that there exists a normal vector field homologous to zero in $N-M$, which in this case would be sufficient to prove the conclusion of Theorem 5.2.

THeOREM 5.3. Let us suppose $M^{4} \subset N^{7}$. If $[M, i]=0$ in $\Omega_{4}(N)$ then $\nu_{M}$ has a cross-section which is nowhere zero.

Proof. There exists $W \subset N \times I$ such that $\partial W=M \times 0 \subset N \times I$ [1].

Let $\nu_{W}$ and $\nu_{M}$ be the normal fiber bundles of $W$ in $N \times I$ and of $M$ in $N$, respectively. We can also suppose that $\nu_{W} \mid M \times 0=\nu_{M}$.

Let us consider $\bar{W} \subset N \times \mathbb{R}$ to be the double of $W$ and let $i: \bar{W} \rightarrow$ $N \times \mathbb{R}$ and $j: N \times \mathbb{R} \rightarrow \bar{W} \rightarrow N \times \mathbb{R}$ be inclusion maps.

Since $i_{*}\left(\mu_{\bar{W}}\right) \subset \operatorname{im} j_{*}$, then $\bar{W}$ has a normal vector field which is nowhere zero in $N \times \mathbb{R}$ up to the 3-skeleton of $\bar{W}$.

Hence, there exists a 2-dimensional oriented vector bundle $\xi$ over $M$ such that $\nu_{M} \mid M^{(3)}=\xi \otimes \mathscr{E}^{1}$.

Let us consider $e$ to be the Euler class of $\xi$ in $H^{2}\left(M^{(3)}\right)$ and let $\bar{e} \in H^{2}(M)$ be such that $i o^{*}(\bar{e})=e$, where $i: M^{(3)} \rightarrow M$ is the inclusion map.

Let $\bar{\xi}$ be a 2-dimensional vector bundle over $M$ such that its Euler class is $\bar{e}$. Let us observe that $\bar{\xi} \mid M^{(3)}=\xi$.

Let $f, g: M \rightarrow \mathrm{BSO}(3)$ be classifying maps $\bar{\xi} \oplus \mathscr{E}^{1}$ and $\nu_{M}$, respectively.

Since the Euler classes of $\bar{\xi} \oplus \mathscr{E}^{1}$ and of $\nu_{M}$ are equal, then their second Stiefel-Whitney classes are equal.

Let $\tilde{p}_{1}$ be the Pontryagin class of the classifying fiber bundle $\tilde{\gamma} \rightarrow$ $\operatorname{BSO}(3)$ and let $\tilde{e}$ be the Euler class of $\tilde{\gamma}$. Since $f^{*}\left(\tilde{p}_{1}\right)=g^{*}\left(\tilde{p}_{1}\right)$. Hence, the vector bundles $\xi \oplus \mathscr{E}^{1}$ and $\nu_{M}$ are equivalent [3]. 


\section{REFERENCES}

[1] C. Biasi, L-equivalent and ambiental bordism of submanifolds, (Doctoral Thesis), ICMSC-USP, São Carlos (1981).

[2] P. E. Conner and E. E. Floyd, Differentiable Periodic Maps, Springer-Verlag (1964).

[3] A. Dold and H. Whitney, Classification of oriented sphere bundles over a 4complex, Ann. of Math., 69 (1959), 667-677.

[4] M. Hirsch, On embedding of bounding manifolds in euclidean space, Ann. of Math., (2), 74 (1961), 494-497.

[5] N. Sato, Cobordism of semiboundary links, Topology Appl., 18 (1984), 225-234.

[6] R. Thom, Quelques propriétés globales de variétés différentiable, Comment. Math. Helv., 28 (1954), 17-86.

Received November 13, 1991 and in revised form November 19, 1992.

ICMSC-USP

Departamento de Matemática

Rua Dr. Carlos Botelho, 1465

Caixa Postal 668

13560-970-SÃo CARLOS-SP-BRASIL 


\section{CONTENTS}

P. Ahern and W. Cohn, Weighted maximal functions and derivatives of invariant Poisson integrals of potentials $\ldots, \ldots, \ldots, \ldots, \ldots, \ldots, \ldots, \ldots, \ldots, \ldots$

V. Ancona, T. Peternell, and J. A. Wiśniewski, Fano bundles and splitting theorems

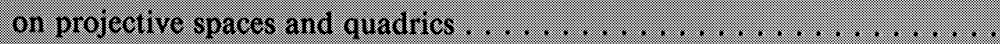

G. M. Beffa, A transverse structure for the Lie-Poisson bracket on the dual of the

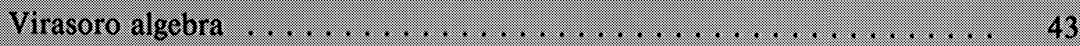

C. Biasi, On ambiental bordism . . . . . . . . . . . . . . . . . 73

P. Debes and M. D. Fried, Nonrigid constructions in Galois theory . . . . . . 81

K. Dykema, Interpolated free group factors . . . . . . . . . . . . . 123

S.-A. Kim and D. Minda, Two-point distortion theorems for univalent functions 137

X. Mo, Value distribution of the Gauss map and the total curvature of complete minimal surface in $R^{m} \ldots \ldots \ldots \ldots \ldots \ldots \ldots \ldots \ldots \ldots$

Z. Shen, On complete Riemannian manifolds with collapsed ends . . . . . . . . 175

F. Zheng, Curvature characterization of certain bounded domains of holomorphy 183

S. Zhu, The classification of complete locally conformally flat manifolds of nonnegative Ricci curvature . . . . . . . . . . . . . . . . . . . . . . 189 


\section{PACIFIC JOURNAL OF MATHEMATICS}

Volume $163 \quad$ No. $1 \quad$ March 1994

Weighted maximal functions and derivatives of invariant Poisson integrals $\quad 1$ of potentials

PATRICK Robert AHERn and WiLliam S. COHN

Fano bundles and splitting theorems on projective spaces and quadrics

VincENZO ANCONA, ThOMAS PETERnELL and JAROSLAW

WISNIEWSKI

A transverse structure for the Lie-Poisson bracket on the dual of the

Virasoro algebra

GLORIA MARI BEFFA

On ambiental bordism

CARLOS BIASI

Nonrigid constructions in Galois theory

PIERRE DÈbes and Michael Fried

Interpolated free group factors

KENNETH JAY DYKEMA

Two-point distortion theorems for univalent functions

SEONG-A KIM and C. DAVID (CARL) MINDA

Value distribution of the Gauss map and the total curvature of complete minimal surface in $R^{m}$

XIAOKANG Mo

On complete Riemannian manifolds with collapsed ends

ZHONGMIN SHEN

Curvature characterization of certain bounded domains of holomorphy

FANGYANG ZHENG

The classification of complete locally conformally flat manifolds of nonnegative Ricci curvature

SHUN-HUi ZHU 